\title{
Epilepsy Treatment Outcome and Its Predictors among Ambulatory Patients with Epilepsy at Mizan-Tepi University Teaching Hospital, Southwest Ethiopia
}

\author{
Ameha Zewudie $\mathbb{D}^{1},{ }^{1}$ Yitagesu Mamo ${ }^{D},{ }^{1}$ Desalegn Feyissa $\left(\mathbb{D},{ }^{1}\right.$ Mohammed Yimam (D), \\ Gosaye Mekonen $\mathbb{}^{2}{ }^{2}$ and Ahmed Abdela ${ }^{3}$ \\ ${ }^{1}$ Department of Pharmacy, College of Medicine and Health Science, Mizan-Tepi University, Mizan-Aman, Ethiopia \\ ${ }^{2}$ Department of Pharmacy, College of Medicine and Health Science, Ambo University, Ambo, Ethiopia \\ ${ }^{3}$ Department of Pharmacy, College of Health Science, Bule-Hora University, Bule-Hora, Ethiopia \\ Correspondence should be addressed to Ameha Zewudie; amehazewudie@gmail.com
}

Received 16 December 2019; Revised 24 February 2020; Accepted 11 March 2020; Published 8 April 2020

Academic Editor: Vincenzo Di Lazzaro

Copyright (c) 2020 Ameha Zewudie et al. This is an open access article distributed under the Creative Commons Attribution License, which permits unrestricted use, distribution, and reproduction in any medium, provided the original work is properly cited.

\begin{abstract}
Background. Epilepsy is among the most common neurological disorders which is highly treatable with currently available antiepileptic drugs at a reasonable price. In Ethiopia, despite a number of studies revealed high prevalence of epilepsy, little is known on predictors of poorly controlled seizures. Thus, the aim of this study was to assess epilepsy treatment outcome and its predictors among patients with epilepsy on follow-up at the ambulatory care unit of Mizan-Tepi University Teaching Hospital, Southwest Ethiopia. Methods. A hospital-based cross-sectional study involving patient interview and chart review was conducted from March 10 to April 10, 2018. Drug use patterns and sociodemographic data of the study participants were accustomed to descriptive statistics. Backward logistic regression analysis was done to identify predictors of poor seizure control. Statistical significance was considered at $p$ value $<0.05$. Results. From a total of 143 studied patients with epilepsy, $60.8 \%$ had uncontrolled seizures. Monotherapy (79\%) was commonly used for the treatment of seizures, of which phenobarbital was the most commonly utilized single anticonvulsant drug (62.9\%). The majority (72.7\%) of the patients had developed one or more antiepileptic-related adverse effects. Medium medication adherence (adjusted odds ratio (AOR) $=5.4 ; 95 \% \mathrm{CI}=1.52-19.23 ; p=0.009$ ), poor medication adherence $(\mathrm{AOR}=8.16 ; 95 \% \mathrm{CI}=3.04-21.90 ; p=0.001)$, head injury before seizure occurrence $(\mathrm{AOR}=4.9 ; 95 \%$ $\mathrm{CI}=1.25-19.27 ; p=0.02$ ), and seizure attacks $\geq 4$ episodes/week before AEDs initiation (AOR $=8.52 ; \% \mathrm{CI}=2.41-13.45$; $p=0.001)$ were the predictors of uncontrolled seizure. Conclusions. Based on our findings, more than half of the patients with epilepsy had poorly controlled seizures. Nonadherence to antiepileptic drugs, high frequency of seizure attack before AEDs initiation, and history of a head injury before the occurrence of seizure were predictors of uncontrolled seizure. Patient medication adherence should be increased by the free access of antiepileptic drugs and attention should be given for the patients with a history of head injury and high frequency of seizure attacks before AEDs initiation.
\end{abstract}

\section{Background}

Epilepsy is a noncommunicable disease of the brain that affects all communities with unequal distribution. About $10 \%$ of the entire world population living a normal life span can expect to have at least one epileptic seizure. There are about 65 million patients with epilepsy worldwide, of whom $80 \%$ are living in developing countries [1]. This could be underestimated because partial seizures are often underdiagnosed in the less developed world. If these patients have been treated appropriately with AEDs, $70 \%$ of them could be seizure-free $[1,2]$.

About $90 \%$ of people with epilepsy in Africa were untreated despite the fact that highly cost-effective treatments were available [3]. Although currently available therapies with AEDs can effectively treat the majority of newly 
diagnosed patients, nonadherence to therapy and inappropriate use of AEDs may significantly affect seizure control [4]. Adequate patient adherence to AEDs, avoiding any seizure triggering factors, and proper patient education about their diseases and management have been implicated for better seizure control [5].

The proportion of deaths that are epilepsy-related may be much higher in Africa than in other regions of the world. In a series of 164 patients with epilepsy recruited from a rural clinic in Tanzania, $67.1 \%$ of them died and the mortality rate was twofold that of the Tanzanian rural population. In greater than $50 \%$ of the patients, the causes of death were related to epilepsy [6].

Epilepsy accounts for $0.6 \%$ of all diseases globally, as a result of years of life lost due to early mortality and time lived with a disability. It has a high economic burden in terms of health care needs, early death, and unproductivity due to absenteeism. A study conducted in India revealed that the cost per patient for epilepsy treatment was as high as $88.2 \%$ of the Indian's per capita gross national product (GNP). This epilepsy-related cost includes travel cost, lost work time, and medical costs which exceeded $\$ 2.6$ billion/year [7].

To solve the problem, the World Health Assembly (WHA) adopted a resolution called the burden of epilepsy globally and at countries level to address its health, public knowledge, and social implications. This resolution advocates governments to formulate, strengthen, and implement their policies to facilitate access to care and protect the right of patients with epilepsy. It focuses on the relevance of training general practitioners and other health care providers in order to reduce epilepsy treatment gaps [7]. Regardless of these interventions, epilepsy is poorly controlled all over the world particularly in developing countries.

To our knowledge, there is no study in Southwest Ethiopia on predictors of poorly controlled seizure. Moreover, studies conducted on determinants of poorly controlled seizures in North Ethiopia had major limitations. In those studies, a controlled seizure was defined as if the patients were seizurefree for 6 months or 1 year. This definition does not fulfill the World Health Organization's (WHO) definition of controlled seizure. In our study, patients with epilepsy were assessed if they were on AEDs for at least 2 years. Seizures are controlled if the patients were seizure-free for at least 2 years. Hence, this study aimed to assess epilepsy treatment outcome and its predictors among patients with epilepsy on follow-up at the ambulatory care unit of Mizan-Tepi University Teaching Hospital (MTUTH), Southwest Ethiopia.

\section{Methods}

2.1. Study Design and Setting. A hospital-based cross-sectional study was conducted in MTUTH which is found in Mizan-Aman Town and located at $561 \mathrm{~km}$ away from the capital city of Ethiopia, Addis Ababa. The study was conducted from March 10 to April 10, 2018.

2.2. Study Participants. The source of the population was all epileptic patients attending the ambulatory unit of MTUTH, while the study population consists of all epileptic patients who were attending the ambulatory unit of MTUTH during the study period and fulfilled inclusion criteria. Patients (age $\geq 15$ years) with a diagnosis of epilepsy who have been taking AEDs and on regular follow-up for at least two years were included in our study. The participants were interviewed, and their medical records were reviewed during their appointment for medication refilling. They were excluded from the study if their medical records were with incomplete data, if they were mentally unstable (such as aggressive patients, critically ill patients, patients with acute psychosis, panic attack, or status epilepticus), or if they were patients who were acute sick looking (determined by clinical presentation) and refused to give consent, and if they were admitted to the hospital during data collection. Among 182 epileptic patients who have been on follow-up at the ambulatory unit of MTUTH, 143 epileptic patients met inclusion criteria. All patients who fulfilled the inclusion criteria were selected as the study participants.

2.3. Data Collection Procedure, Variables, and Outcome of the Study. All epileptic patients who have been on follow-up at the hospital during the data collection period and fulfill inclusion criteria were included in the study. Data abstraction format was used to retrieve patients' clinical information and medication experience such as the status of treatment outcome, types of epilepsy, seizure frequency, duration of follow-up, comorbidity, types of AEDs, and AEDs-related adverse effects from patients' medical record. A semistructured questionnaire was used to collect patients' sociodemographic data, history of head injury, triggering factors, and medication adherence. Medication adherence was assessed using a self-reported questionnaire which was developed based on the review of literatures [8-11]. Patients were asked whether or not they had missed or stopped a dose of their AED for any reason in the last one month. We assessed the adherence level in the last one-month period because of published literatures supporting the one-month recall period $[8,12,13]$. Accordingly, epileptic patients who took their AEDs without missing any dose in the last month were considered as high adherence. Epileptic patients who missed 1 dose of their AEDs in the last month were said to be medium adherence, whereas patients who missed 2 or more doses were considered as low adherence to AEDs.

Since we have no data on definite diagnosis for the type of epilepsy except generalized tonic-clonic seizure, and genetic variability between the participants, these variables can be considered as potential confounders. Epilepsy treatment outcome was assessed in terms of seizure control and seizure frequency. The seizure status was considered to be well controlled if the patients had not experienced any seizure episode in the last two years, and poorly controlled if they experienced one or more seizure episodes in the last two years of follow-up period.

2.4. Statistical Analysis. The data were entered into Epidata manager version 4.0.2.101 and analyzed by Statistical Package for Social Science (SPSS) version 21. Bivariate logistic 
regression was done to see the association between independent variables and seizure control. Variables with $p$ value $\leq 0.25$ on bivariate logistic regression were entered into multivariate logistic regression. Multivariable logistic regression analyses using backward selection were done to identify predictors of poor seizure control at $p$ value of $<0.05$ significant.

\section{Result}

3.1. Sociodemographic Characteristics of the Study Subjects. There were 182 ambulatory epileptic patients at the epilepsy unit of MTUTH. Of them, 9 patients refused an interview, 17 patients were under 15 years, and 13 patients' medical charts were incomplete. One hundred and forty-three participants were interviewed, their medical records were reviewed, and analysis was done. Among the participants, $48.3 \%$ were female. Most patients (86.8\%) were below 45 years of age and more than half $(54.6 \%)$ of the patients' ages fell between 15 and 30 years. More than half (55.2\%) of the participants attended primary school and about $37.1 \%$ of epileptic patients were students (Table 1).

3.2. Clinical Information and Determinants of Prognostic Factors. Among the studied participants, 39.1\% were seizurefree while $60.8 \%$ of them had one or more seizure episodes during the last 2 years. Generalized tonic-clonic seizure (GTCS) was the most commonly diagnosed type of epilepsy (77.6\%). About $80.5 \%$ of studied participants had 2-5 years of follow-up period and $28.0 \%$ had one or more comorbidities. A psychiatric disorder was (13.3\%) the most commonly identified comorbidity. About $73.4 \%$ of the studied patients with epilepsy had one or more seizure precipitating factors and less than half $(44.0 \%)$ of them suffered from emotional stress as a seizure triggering factor. Among the studied participants, $47.5 \%$ had a history of brain injury; of them, $37.9 \%$ had a brain injury before seizure occurrence (Table 2).

3.3. Medication Experience of Epileptic Patients. The initial AEDs prescribed for patients with epilepsy were phenobarbitone $(81.8 \%)$, phenytoin $(15.42 \%)$, and carbamazepine (2.88\%). Among AEDs, the most commonly used drug as add-on therapy for the patients whose seizure was not controlled was phenytoin (14.7\%). Phenobarbital was prescribed for $81.8 \%$ of the participants and $21 \%$ of the patients had one or more antiepileptic drugs. The majority $(72.7 \%)$ of the patients with epilepsy had developed one or more antiepileptic-related adverse effects. More than half (54.5\%) of the studied participants had developed sedation. The other AEDs-related adverse effects were confusion (7.0\%), weakness $(6.3 \%)$, gingival hyperplasia $(6.3 \%)$, rash $(4.9 \%)$, blurred vision $(4.2 \%)$, and gastrointestinal irritation (GI) (2.8\%) (Table 3).

3.4. Adherence Status and Reasons for Nonadherence. Among 143 studied participants, 58.7\%, 16.8\%, and 24.5\% had low, medium, and high adherence to AEDs, respectively. The most common reasons for nonadherence were
TABLE 1: Sociodemographic characteristics of epileptic patients on AEDs at MTUTH from March 10 to April 10, 2018.

\begin{tabular}{|c|c|}
\hline Sociodemographic characteristic & Frequency $(N=143)$ \\
\hline \multicolumn{2}{|l|}{ Age } \\
\hline $15-30$ & $78(54.6)$ \\
\hline $31-45$ & $46(32.2)$ \\
\hline 46 and above & $1913.3)$ \\
\hline \multicolumn{2}{|l|}{ Sex } \\
\hline Male & $74(51.7)$ \\
\hline Female & $69(48.3)$ \\
\hline \multicolumn{2}{|l|}{ Marital status } \\
\hline Married & $71(49.7)$ \\
\hline Single & $63(44.1)$ \\
\hline Divorced & $4(2.8)$ \\
\hline Widowed & $5(3.5)$ \\
\hline \multicolumn{2}{|l|}{ Occupation } \\
\hline Government employee & $17(11.9)$ \\
\hline Farmers & $41(28.7)$ \\
\hline Students & $53(37.1)$ \\
\hline Merchants & $24(16.8)$ \\
\hline Daily labors & $8(5.6)$ \\
\hline \multicolumn{2}{|l|}{ Place of residence } \\
\hline Rural & $88(61.5)$ \\
\hline Urban & $55(38.5)$ \\
\hline \multicolumn{2}{|l|}{ Level of education } \\
\hline Not educated & $15(10.5)$ \\
\hline Primary $(1-8)$ & $79(55.2)$ \\
\hline Secondary (9-12) & $27(18.9)$ \\
\hline College/university & $22(15.4)$ \\
\hline \multicolumn{2}{|l|}{ Monthly income } \\
\hline Less than 500 & $35(24.5)$ \\
\hline $500-1000$ & $51(35.7)$ \\
\hline 1000-2000 & $43(30.1)$ \\
\hline 2000 and above & $14(9.7)$ \\
\hline
\end{tabular}

forgetfulness (43.5\%) and unaffordability (40.7\%). Lack of education about AEDs (31.5\%), long distance from treatment setting (27.8\%), unscheduled hospital follow-up due to workload $(21.3 \%)$, high cost of medication (19.4\%), and other less common factors were reported by the patients (Table 4).

3.5. Predictors of Poor Treatment Outcome. All variables with $p$ value $<0.25$ in bivariate logistic regression were entered into multivariate logistic regression to control confounding variables. After adjusting for the other variables, medium adherence to $\mathrm{AEDs} \quad(\mathrm{AOR}=5.40 ; \quad 95 \% \quad \mathrm{CI}=1.52-19.23$; $p=0.009$ ), low adherence to AEDs (AOR $=8.16 ; 95 \%$ $\mathrm{CI}=3.04-21.90 ; p=0.001)$, history of head injury before seizure occurrence $(\mathrm{AOR}=4.90 ; 95 \% \quad \mathrm{CI}=1.25-19.27$; $p=0.02)$, and seizure attack $>4$ episodes/week $(\mathrm{AOR}=1.98$; 95\% CI $=1.053-5.978 ; p=0.012$ ) before AEDs initiation were independent predictors of poorly controlled seizure (Table 5).

\section{Discussion}

The current study aimed to assess epilepsy treatment outcome and its predictors among patients with epilepsy who had been on follow-up at the ambulatory clinic of MTUTH, Southwest Ethiopia. 
TABLE 2: Clinical information and seizure treatment outcome among epileptic patients on AEDs at MTUTH from March 10 to April 10, 2018.

\begin{tabular}{|c|c|}
\hline $\begin{array}{l}\text { Clinical information and determinants of } \\
\text { prognostic factors }\end{array}$ & $\begin{array}{l}\text { Frequency } \\
(\%)\end{array}$ \\
\hline $\begin{array}{l}\text { Status of treatment outcome } \\
\text { Well-controlled } \\
\text { Poorly controlled }\end{array}$ & $\begin{array}{l}56(39.2) \\
87(60.8)\end{array}$ \\
\hline $\begin{array}{l}\text { Types of epilepsy } \\
\text { Generalized tonic-clonic seizure } \\
\text { Unclassified epilepsy }\end{array}$ & $\begin{array}{c}111(77.6) \\
32(22.4) \\
\end{array}$ \\
\hline $\begin{array}{l}\text { Time on AEDs } \\
2-5 \text { years } \\
5 \text { years and above } \\
\end{array}$ & $\begin{array}{c}120(83.9) \\
23(16.1) \\
\end{array}$ \\
\hline $\begin{array}{l}\text { Follow-up in the clinic } \\
2-5 \text { years } \\
5 \text { years and above }\end{array}$ & $\begin{array}{c}115(80.5) \\
28(19.6)\end{array}$ \\
\hline $\begin{array}{l}\text { Frequency of seizure attack/week before AEDs } \\
\text { initiation } \\
\quad<4 \\
\geq 4\end{array}$ & $\begin{array}{l}69(48.2) \\
74(52.8) \\
\end{array}$ \\
\hline $\begin{array}{l}\text { Comorbidity } \\
\text { Yes } \\
\text { No } \\
\end{array}$ & $\begin{array}{c}40(28.0) \\
103(72.0) \\
\end{array}$ \\
\hline $\begin{array}{l}\text { Types of comorbidity } \\
\text { Psychiatric conditions } \\
\text { Hypertension } \\
\text { Diabetic mellitus } \\
\text { Heart failure } \\
\end{array}$ & $\begin{array}{c}19(13.3) \\
11(7.7) \\
7(4.9) \\
3(2.1) \\
\end{array}$ \\
\hline $\begin{array}{l}\text { Triggering factors } \\
\text { Yes } \\
\text { No }\end{array}$ & $\begin{array}{c}105(73.4) \\
38(26.6)\end{array}$ \\
\hline $\begin{array}{l}\text { Types of triggering factors } \\
\text { Emotional stress } \\
\text { Sleep deprivation } \\
\text { Missing medication } \\
\text { Heavy alcohol use } \\
\text { Chat and stimulant } \\
\text { Others }\end{array}$ & $\begin{aligned} & 63(44.0) \\
& 37(25.9) \\
& 23(16.1) \\
& 5(3.9) \\
& 4(2.8) \\
& 9(6.3) \\
&\end{aligned}$ \\
\hline $\begin{array}{l}\text { Time since seizure-free } \\
2-5 \text { yrs } \\
5 \text { and above }\end{array}$ & $\begin{array}{c}47(32.9) \\
9(6.3)\end{array}$ \\
\hline $\begin{array}{l}\text { Time to enter remission phase } \\
2-5 \text { yrs } \\
5 \text { and above }\end{array}$ & $\begin{array}{l}39(27.3) \\
17(11.9) \\
\end{array}$ \\
\hline $\begin{array}{l}\text { History of head injury } \\
\text { Yes } \\
\text { No }\end{array}$ & $\begin{array}{l}68(47.5) \\
75(52.4) \\
\end{array}$ \\
\hline $\begin{array}{l}\text { Time of head injury } \\
\text { Before seizure occurrence } \\
\text { After seizure occurrence } \\
\end{array}$ & $\begin{array}{l}53(37.0) \\
15(10.5) \\
\end{array}$ \\
\hline $\begin{array}{l}\text { EEG abnormality } \\
\text { Yes } \\
\text { No }\end{array}$ & $\begin{array}{l}90 \\
53 \\
\end{array}$ \\
\hline
\end{tabular}

Others: dust, anger, high temperature, and headache. ECG: electrocardiogram.

This study revealed that the most common type of seizure was GTCS. The reason for this might be multifactorial. Knowing the two main semiological components of seizures (physiologic and autonomic) is the basic step in exactly diagnosing the type of seizures. For instance, GTCS may present with violent body movements and often prominent autonomic changes. As a result, the health care seeking in those populations is higher than other types of seizures [14]. This argument is also supported by a study conducted in Ethiopia which showed that $95.1 \%$ of patients with epilepsy seek treatment if there is a sudden loss of consciousness [15]. In contrast to this, the partial or focal seizure had been often underdiagnosed in a developing country, because of different reasons [1, 2]. For instance, unlike that of GTCS, in focal seizure, disturbance of cognition is inapparent and loss of consciousness is not usual [14]. Even though traumatic brain injury is a known cause of focal/partial seizure, most of the symptoms associated with simple partial seizure are internal and only noticed by the person having the seizure, another hindering factor for clinical diagnosis and for seeking health care [16]. Thus, such type of seizure may be considered as an unclassified type of seizure. Therefore, these might be the reasons for why GTCS was the most common type of seizure compared to the other seizure types in our study.

In this study, the most commonly prescribed AED as monotherapy was phenobarbitone $(81.8 \%)$ followed by phenytoin (15.4\%). This is in line with the studies conducted in Jimma and Gonder, Ethiopia $[17,18]$. In contrast to this, a study done in the United Kingdom reported that the most commonly used AED was carbamazepine (37.4\%) followed by sodium valproate (35.7\%) [19]. This might be due to the difference in sociodemographic characteristics of the patients and a large sample size used in the previous study.

The majority of the patients were on monotherapy and only one-fourth of them were on polytherapy. The most commonly used AEDs as polytherapy were the combination of phenobarbitone with phenytoin (11.9\%) followed by the combination of the phenobarbitone and carbamazepine (6.3\%). In contrast to this, studies done in different parts of India revealed that either phenytoin or carbamazepine was the most prescribed AED as monotherapy [20, 21].

Although optimum AED therapy eliminates seizure by $70 \%$, approximately one-third of patients continue to experience seizures despite appropriate treatment [22, 23]. But, our finding was contrary to these reports; only $39 \%$ of patients with epilepsy were seizure-free during the last two years. This figure is lower as compared with the study done in different parts of the country $[18,21]$. The reason could be the difference in the duration of the outcome measurement report, which was short in the previous study. For instance, the study done in Gonder and Mekele measured the treatment outcome after the patients took AEDs for 3 months and 1 year, respectively $[18,24]$. But, in the current study, the status of seizure was measured for the epileptic patients on AEDs for at least 24 months. The second reason might be level of adherence. Studies showed that $70 \%$ of controlled seizure was reported for patients with optimum or good adherence level to AEDs [22, 23]. In our study, majority of the patients were nonadherent to AEDs.

In this study, the most commonly diagnosed comorbidity among studied patients with epilepsy was found to be 
TABLE 3: Antiepileptic drugs and associated adverse effects among epileptic patients on AEDs at MTUTH from March 10 to April 10, 2018.

\begin{tabular}{|c|c|c|}
\hline Antiepileptic drug & Adverse effects & Frequency (\%) \\
\hline \multirow{5}{*}{ Types of AEDs used } & Phenobarbitone alone & $90(62.9)$ \\
\hline & Phenytoin alone & $23(16.1)$ \\
\hline & Phenobarbitone plus phenytoin & $17(11.9)$ \\
\hline & Phenobarbitone plus carbamazepine & $9(6.3)$ \\
\hline & Carbamazepine plus phenytoin & $4(2.8)$ \\
\hline \multirow{3}{*}{ AEDs prescribed as initial therapy } & Phenobarbitone & $117(81.8)$ \\
\hline & Phenytoin & $22(15.4)$ \\
\hline & Carbamazepine & $4(2.8)$ \\
\hline \multirow{2}{*}{ AEDs prescribed as add-on therapy } & Phenytoin & $21(14.7)$ \\
\hline & Carbamazepine & $12(8.4)$ \\
\hline \multirow{2}{*}{ Polytherapy with AEDs } & Yes & $30(21.0)$ \\
\hline & No & $113(79.0)$ \\
\hline \multirow{2}{*}{ Polytherapy irrespective of AEDs } & Yes & $70(48.9)$ \\
\hline & No & $73(51.1)$ \\
\hline \multirow{2}{*}{ Antiepileptic-related adverse effects } & Yes & $104(72.7)$ \\
\hline & No & $39(27.3)$ \\
\hline \multirow{7}{*}{ Types of antiepileptic associated adverse effects } & Sedation & $78(54.5 \%)$ \\
\hline & Confusion & $10(7.0)$ \\
\hline & Weakness & $9(6.3)$ \\
\hline & Gingival hyperplasia & $9(6.3)$ \\
\hline & Rash & $7(4.9)$ \\
\hline & Blurred vision & $6(4.2)$ \\
\hline & GI irritation & $4(2.8)$ \\
\hline
\end{tabular}

TABLE 4: Reasons for nonadherence among epileptic patients on AEDs at MTUTH from March 10 to April 10, 2018.

\begin{tabular}{lccc}
\hline Reasons for no adherence & Reasons for nonadherence & Frequency & Percent \\
\hline & You cannot afford to buy the medications & 44 & 40.7 \\
Patient-related factors & The schedule of your work makes it impossible & 23 & 21.3 \\
& Forget to take medications & 11 & 10.2 \\
Medication-related factor & Medication-related side effect & 14.8 \\
& Misunderstanding of instructions about how to take the drugs & 11 & 6 \\
Health system-related factor & Lack of free medicine supply & 34 & 8 \\
& Lack of education about AEDs & 31.5 \\
& Poor relationship between patient and physician & 7.4 & 43.5 \\
Condition-related factors & Forgetfulness & 6.5 \\
& Memory deficits & 3 & 2.8 \\
Socioeconomic-related factors & High frequency of seizure & 1 & 3.93 \\
& Duration and previous treatment failure & 27.8 \\
\end{tabular}

a psychiatric condition (13.3\%). This is similar to the study done in the USA (33.7\%) and Mekelle, Ethiopia (20.37\%) where the psychiatric condition was the most common comorbidity [24, 25].

In the present study, the majority of the patients had developed AEDs-related adverse effects (72.7\%). This is higher than the studies done in Mekelle and Ambo, Ethiopia, where $43.3 \%$ and $41.7 \%$ of patients experienced AEDs-related adverse effects, respectively [24, 26]. The discrepancy might be due to the fact that, in the current study, some of the patients were diagnosed with an unclassified type of seizures which might result in incorrect dose, frequency, drug selection, and drug interaction which in turn cause adverse drug effects. The other probable reason might be due to the fact that, in our study, the studied participants were exposed to AEDs for the duration of $\geq 2$ years whereas $\leq 1$ year in the previous studies.

Our findings indicated that level of adherence, history of a head injury before seizure occurrence, and frequency of seizure attacks before AEDs initiation were found to be determinant factors of epilepsy treatment outcome. The result of this study showed that low medication adherence was an independent predictor of uncontrolled seizures. This is in line with the studies done in Gonder and Mekelle, Ethiopia [18, 24]. In addition, this study showed that high adherence to AEDs decreases the risk of seizure attacks [27]. In this study, the most common reason for nonadherence was forgetfulness (43.5\%). In a similar way, the study done in China indicated that $69.6 \%$ of the participants had forgotten to take their AEDs [23]. Moreover, a study conducted in 
TABLE 5: Predictors of poorly controlled seizure among epileptic patients on AEDs at MTUTH from March 10 to April 10, 2018.

\begin{tabular}{|c|c|c|c|c|}
\hline \multirow[b]{2}{*}{ Variables affecting treatment outcome } & \multicolumn{2}{|c|}{ Seizure treatment outcome } & \multirow[b]{2}{*}{ AOR $(95 \% \mathrm{CI})$} & \multirow[b]{2}{*}{$p$ value } \\
\hline & $\begin{array}{l}\text { Controlled frequency } \\
\qquad(\%)\end{array}$ & $\begin{array}{l}\text { Noncontrolled frequency } \\
\qquad(\%)\end{array}$ & & \\
\hline \multicolumn{5}{|l|}{ Level of adherence } \\
\hline High adherence & $25(71.4 \%)$ & $10(28.6 \%)$ & 1.00 & 1.00 \\
\hline Medium adherence & $12(50 \%)$ & $12(50 \%)$ & $5.4(1.52-19.23)$ & 0.009 \\
\hline Low adherence & $19(22.6 \%)$ & $65(77.4 \%)$ & $\begin{array}{c}8.16 \\
(3.04-21.90) \\
\end{array}$ & 0.001 \\
\hline \multicolumn{5}{|l|}{ History of head injury } \\
\hline Yes (68) & $19(28 \%)$ & $49(72 \%)$ & $\begin{array}{c}2.4 \\
(1.088-5.314)\end{array}$ & 0.03 \\
\hline No (75) & $37(49.3 \%)$ & $38(50.7)$ & 1.00 & 1.00 \\
\hline \multicolumn{5}{|l|}{ History of head injury } \\
\hline Before seizure occurrence & $10(18.9 \%)$ & $43(81.1 \%)$ & $\begin{array}{c}4.90 \\
(1.25-19.27)\end{array}$ & 0.02 \\
\hline After seizure occurrence & $9(60 \%)$ & $6(40 \%)$ & 1.00 & 1.00 \\
\hline \multicolumn{5}{|c|}{$\begin{array}{l}\text { Frequency of seizure attacks per week before AEDs } \\
\text { initiation }\end{array}$} \\
\hline$<3$ times & $43(62.3 \%)$ & $26(37.7 \%)$ & 1.00 & 1.00 \\
\hline$>4$ times & $15(20 \%)$ & $59(79.7 \%)$ & $1.98(1.055 .98)$ & 0.012 \\
\hline \multicolumn{5}{|l|}{ Marital status } \\
\hline Married & $26(36.6 \%)$ & $45(63.4 \%)$ & 1.00 & 1.00 \\
\hline Single & $26(41.3 \%)$ & $37(58.7 \%)$ & $0.64(0.27-1.51)$ & 0.31 \\
\hline Divorced & 0 & 4 & $9.9(0.05-4.45)$ & 0.99 \\
\hline Widowed & $4(80 \%)$ & $1(20 \%)$ & $0.08(0.01-0.93)$ & 0.04 \\
\hline \multicolumn{5}{|l|}{ Monthly income } \\
\hline$>2000$ & $8(57.1 \%)$ & $6(42.9 \%$ & 1.00 & 1.00 \\
\hline $1000-2000$ & $15(34.9 \%)$ & $28(65.1 \%)$ & $2.09(0.71-7.51)$ & 0.246 \\
\hline $500-1000$ & $15(48.4 \%)$ & $16(51.6 \%)$ & $1.62(0.29-4.57)$ & 0.384 \\
\hline Less than 500 & $9(36 \%)$ & $16(64 \%)$ & $2.03(0.32-1.99)$ & 0.236 \\
\hline \multicolumn{5}{|l|}{ Educational status } \\
\hline College/university & $7(31.8 \%)$ & $15(68.2 \%)$ & 1.00 & 1.00 \\
\hline Secondary $(9-12)$ & $10(37 \%)$ & $17(63)$ & $1.05(0.12-1.86)$ & 0.583 \\
\hline Primary $(1-8)$ & $31(39.2 \%)$ & $48(60.8 \%)$ & $2.20(0.65-2.87)$ & 0.526 \\
\hline Not educated & $8(53.3 \%)$ & $7(46.7 \%)$ & $1.02(0.11-1.58)$ & 0.095 \\
\hline
\end{tabular}

Ethiopia previously revealed that forgetfulness was the main reason for nonadherence [28].

Even though the majority of our study participants have been taking phenobarbital, AED with long duration of action, it was found that missing one dose per month (medium medication adherence) was an independent predictor of poor seizure control. The possible reason that justifies the association might be self-reporting-related bias. Study indicated that underreporting of adherence level (selfreporting-related bias) and trying to reduce number of missed medications to get acceptance from health care providers is the main problem of measuring adherence [29].

Studies revealed that uncontrolled seizure was more likely among individuals who had a history of head injury than those who had no history of a head injury before seizure occurrence $[18,30]$. Similarly, head injury before diagnoses of epilepsy was found to be the determinant of uncontrolled seizures in this study. Since this study is a cross-sectional study, it cannot address the causal effect. Study indicates that seizures are a common complication of head injuries [6]. In contrast to the current study, a study conducted in Ambo, Ethiopia, showed that there is no significant association between head injury and uncontrolled seizure [26].
In our study, a high frequency of seizure episodes (greater than or equal to four seizure attacks per week before AED initiation) was the other independent predictor of uncontrolled seizures. To our knowledge, there is only one study researched with the variable "number of seizure attacks per week before AEDs initiation" as an independent variable. In this study, there is an association between the number of seizure attacks per week before AEDs initiation and uncontrolled seizure on bivariate analysis. But the author did not run multivariate analysis to identify independent predictors of the uncontrolled seizure [26].

Finally, our study is not without limitations. First, the cross-sectional nature of the study may not provide adequate evidence of causality between poor seizure control and predictor variables. Second, due to self-report concerns, patients may understate socially undesirable activities like medication nonadherence. Lastly, our findings cannot be generalized to the whole patients with epilepsy found in Southwest Ethiopia because of two reasons. First, the study showed that about $54.6 \%$ of patients with epilepsy found in rural Ethiopia have poor treatment-seeking behavior because of social stigma, lack of knowledge, unfavorable attitude, and lack of social support [31]. Another study 
conducted in Ethiopia showed about $95.1 \%$ of patients with epilepsy seek treatment if there is a sudden loss of consciousness [15]. Second, our study was conducted in a single hospital found in Southwest Ethiopia.

\section{Conclusions}

Based on our study, more than half of the patients with epilepsy had uncontrolled seizures. Nonadherence to AEDs, number of seizure attacks before AEDs initiation, and head injury before seizure occurrence were predictors of uncontrolled seizure. AEDs adherence should be increased by an access to antiepileptic drugs without charge and attention should be given to patients with a history of head injury and the high number of seizure attacks/week before AEDs initiation. Moreover, we recommend researchers to do further longitudinal and interventional studies to provide adequate evidence about the cause-effect relationship between the predictor variables and seizure control.

\section{Abbreviations}

AOR: $\quad$ Adjusted odds ratio

AEDs: Antiepileptic drugs

GTCS: Generalized tonic-clonic seizure

GNP: Gross national product

MTUTH: Mizan-Tepi University Teaching Hospital

SPSS: $\quad$ Statistical Package for Social Science

WHA: World Health Assembly

WHO: World Health Organization.

\section{Data Availability}

The raw data supporting the results reported in this article are available upon reasonable request by contacting the corresponding author AZ.

\section{Ethical Approval}

Ethical clearance was obtained from the Ethical Review Board of Mizan-Tepi University, College of Medicine and Health Science.

\section{Consent}

Written informed consent for participation in the study was obtained from the patients or from their parent or guardian if participants are children under 16 years of age. The confidentiality of all the patients was maintained.

\section{Conflicts of Interest}

The authors declare that they have no conflicts of interest.

\section{Authors' Contributions}

AZ made substantial contributions to conception, design of the work, methodology, analysis, and data interpretation, wrote the final manuscript, and managed the overall progress of the study. YM, DF, GM, and MY helped providing data, interpreted data, and analyzed and checked the final version of the article. AA conceptualized the study design, analyzed and interpreted the data, and wrote and revised the article. All authors read and approved the final manuscript.

\section{Acknowledgments}

The authors would like to thank the data collectors and study participants.

\section{References}

[1] World Health Organization, Epilepsy: Epidemiology, Etiology and Prognosis, World Health Organization, Geneva, Switzerland, 2001.

[2] M. Leonardi and T. B. Ustun, "The global burden of epilepsy," Epilepsia, vol. 43, no. 6, pp. 21-25, 2002.

[3] World Health Organization, The Global Burden of Mental and Neurological Problems, WHO, Geneva, Switzerland, 1999.

[4] R. M. Jones, J. A. Butler, V. A. Thomas, R. C. Peveler, and M. Prevett, "Adherence to treatment in patients with epilepsy: associations with seizure control and illness beliefs," Seizure, vol. 15, no. 7, pp. 504-508, 2006.

[5] I. E. Leppik, "How to get patients with epilepsy to take their medication," Postgraduate Medicine, vol. 88, no. 1, pp. 253256, 1990.

[6] A. G. Diop, D. C. Hesdorffer, G. Logroscino, and W. A. Hauser, "Epilepsy and mortality in Africa: a review of the literature," Epilepsia, vol. 46, no. 11, pp. 33-35, 2005.

[7] I. Megiddo, A. Colson, D. Chisholm, T. Dua, A. Nandi, and R. Laxminarayan, "Health and economic benefits of public financing of epilepsy treatment in India: an agent-based simulation model," Epilepsia, vol. 57, no. 3, pp. 464-474, 2016.

[8] C. A. Hovinga, M. R. Asato, R. Manjunath et al., "Association of non-adherence to antiepileptic drugs and seizures, quality of life, and productivity: survey of patients with epilepsy and physicians," Epilepsy \& Behavior, vol. 13, no. 2, pp. 316-322, 2008.

[9] W. Y. Lam and P. Fresco, "Medication adherence measures: an overview," BioMed Research International, vol. 2015, Article ID 217047, 12 pages, 2015.

[10] K. Thompson, J. Kulkarni, and A. A. Sergejew, "Reliability and validity of a new medication adherence rating scale (MARS) for the psychoses," Schizophrenia Research, vol. 42, no. 3, pp. 241-247, 2000.

[11] L. Fialko, P. A. Garety, E. Kuipers et al., "A large-scale validation study of the medication adherence rating scale (MARS)," Schizophrenia Research, vol. 100, no. 1-3, pp. 53-59, 2008.

[12] P. Doró, R. Benkő, A. Czakó, M. Matuz, F. Thurzó, and G. Soós, "Optimal recall period in assessing the adherence to antihypertensive therapy: a pilot study," International Journal of Clinical Pharmacy, vol. 33, no. 4, pp. 690-695, 2011.

[13] J. Doughty, G. A. Baker, A. Jacoby, and V. Lavaud, "Compliance and satisfaction with switching from an immediate-release to sustained-release formulation of valproate in people with epilepsy," Epilepsy \& Behavior, vol. 4, no. 6, pp. 710-716, 2003.

[14] A. Ulate-Campos, F. Coughlin, M. Gaínza-Lein, I. S. Fernández, P. Pearl, and T. Loddenkemper, "Automated seizure detection systems and their effectiveness for each type of seizure," Seizure, vol. 40, pp. 88-101, 2014.

[15] B. B. Bifftu, B. A. Dachew, B. T. Tiruneh, and W. G. Alemu, "First choice of treatment place in the pathways to epileptic care 
at the outpatient department of University of Gondar Hospital, Northwest Ethiopia: cross-sectional institutional based study," PLoS One, vol. 12, no. 8, Article ID e0181310, 2017.

[16] J. Englander, D. X. Cifu, and R. Diaz-Arrastia, "Seizures and traumatic brain injury," Archives of Physical Medicine and Rehabilitation, vol. 95, no. 6, pp. 1223-1224, 2014.

[17] M. Gurshaw, A. Agalu, and T. Chanie, "Anti-epileptic drug utilization and treatment outcome among epileptic patients on follow-up in a resource poor setting," Journal of Young Pharmacists, vol. 6, no. 3, pp. 47-52, 2014.

[18] M. Tigistu, T. Azale, H. Kebebe, and T. Yihunie, "Frequency of seizure attack and associated factors among patients with epilepsy at university of Gondar referral hospital: a crosssectional study, Gondar, North West Ethiopia," BMC Research Note, vol. 11, no. 1, pp. 3761-3763, 2018.

[19] N. F. Moran, K. Poole, G. Bell et al., "Epilepsy in the United Kingdom: seizure frequency and severity, anti-epileptic drug utilization and impact on life in 1652 people with epilepsy," Seizure, vol. 13, no. 6, pp. 425-433, 2004.

[20] R. Gurumurthy, K. Chanda, and G. Sarma, "An evaluation of factors affecting adherence to antiepileptic drugs in patients with epilepsy: a cross-sectional study," Singapore Medical Journal, vol. 58, no. 2, pp. 98-102, 2017.

[21] Y. P. Mohammed, B. Debdipta, M. Sushma, B. Jagadish, and Agadi, "Assessment of factors influencing self reported drug adherence to antiepileptic drugs at a tertiary care hospital," International Journal of Research in Pharmacology and Pharmacotherapeutics, vol. 6, no. 2, pp. 163-169, 2017.

[22] J. W. Sander, "The use of antiepileptic drugs-principles and practice," Epilepsia, vol. 45, no. 6, pp. 28-34, 2004.

[23] A. C. F. Hui, A. Wong, H. C. Wong, B. L. Man, K. M. AuYeung, and K. S. Wong, "Refractory epilepsy in a Chinese population," Clinical Neurology and Neurosurgery, vol. 109, no. 8, pp. 672-675, 2007.

[24] Y. L. Niriayo, A. Mamo, T. D. Kassa et al., "Treatment outcome and associated factors among patients with epilepsy," Scientific Reports, vol. 8, no. 1, pp. 35906-35912, 2018.

[25] J. P. Szaflarski, A. Y. Rackley, C. J. Lindsell, M. Szaflarski, and S. L. Yates, "Seizure control in patients with epilepsy: the physician vs. medication factors," BMC Health Services Research, vol. 8, no. 1, pp. 6963-6968, 2008.

[26] G. Tefera, T. Woldehaimanot, and M. Angamo, "Poor treatment outcomes and associated factors among epileptic patients at Ambo Hospital, Ethiopia," Gaziantep Medical Journal, vol. 21, no. 1, pp. 9-16, 2015.

[27] R. Manjunath, K. L. Davis, S. D. Candrilli, and A. B. Ettinger, "Association of antiepileptic drug nonadherence with risk of seizures in adults with epilepsy," Epilepsy \& Behavior, vol. 14, no. 2, pp. 372-378, 2009.

[28] D. Dugassa, "Assessment of factors associated to poor treatment outcomes among epileptic patients taking anti epileptic medications at Shambu hospital, Northwest Ethiopia," World Journal of Advance Healthcare Research, vol. 1, no. 2, pp. 1-18, 2017.

[29] M. J. Stirratt, J. Dunbar-Jacob, H. M. Crane et al., "Self-report measures of medication adherence behavior: recommendations on optimal use," Translational Behavioral Medicine, vol. 5, no. 4, pp. 470-482, 2015.

[30] D. H. Lowenstein, "Epilepsy after head injury: an overview," Epilepsia, vol. 50, no. 2, pp. 4-9, 2009.

[31] A. Molla, B. Mekuriaw, E. Habtamu, and M. Mareg, "Treatment-seeking behavior towards epilepsy among rural residents Ethiopia: a cross-sectional study," Neuropsychiatric Disease and Treatment, vol. 16, pp. 433-439, 2020. 